\title{
GH Baltuch and J-G Villemure: Operative techniques in epilepsy surgery
}

\author{
Thieme 2009, ISBN 978-1-60406-030-0
}

\author{
Jens Lehmberg
}

Published online: 11 March 2010

(C) Springer-Verlag 2010

The preface states: “ ..., we endeavoured to contribute a techniques text that would be readable by neurosurgeons and neurosurgeons in training, who want to learn how to perform the classic resective procedures of epilepsy surgery and some of the more recent and investigational neuromodulatory approaches." Did the authors accomplish these aims?

This hardback with letter format is printed on high quality paper. The book includes 127 figures. A mix of drawings, operative and cadaver photographs, radiologic images, and tables illustrations, MR, CT and navigation imaging as well as color intraoperative photographs and an appropriate portion of text on 206 pages. The book is originally written in English. Five sections comprise 20 chapters. 33 authors contributed to this book. The index is highly expedient and consists of ten pages, expressions are going down to four levels. Each chapter ends with a reference list.

The book is divided into five sections. The first is surgical planning including image guidance as well as placement of invasive electrodes for electroencephalography. The second section, titled cortical resection, covers not only amygdalohippocampectomy and lobar resection but also resection of hypothalamic hamartomas and subpial transections. The section intraoperative mapping includes

J. Lehmberg $(\bowtie)$

Oberarzt der Klinik für Neurochirurgie, Klinikum rechts der Isar, Technische Universität,

München Ismaninger Strasse 22,

81675 Munich, Germany

e-mail: Jens.Lehmberg@1rz.tu-muenchen.de en plus callosotomy and hemispherectomy. The neuromodulation section covers the standard vagus stimulation and the investigative procedures of hippocampus, deep brain and responsive stimulation. Last but not least, the radiosurgery chapter forms its own section. The authors state their own technical preferences, which is legitimate, since the authors are all acknowledged experts in their field. At times, the technical steps are described in detail. For example, it seems questionable, whether a sample operating room layout should be comprised.

As with many multi-authored books, there is heterogeneity between the chapters. Chapters like the one about selective amygdalohippocampectomy or functional hemispherectomy are so precise and well illustrated, that one could start treating patient after working through the chapter. One chapter has only six references, other references are very detailed and seem to be too specific. Most striking is, that the introductions of each chapter are at times redundant. Authors delineate here the indications for or the history of epilepsy surgery. And these are the chapters or even the sections that are missing: indication for surgery in general and each procedure in particular and history of epilepsy surgery.

The authors do indeed accomplish their aims. The book gives an up to date overview of the techniques in epilepsy surgery. It comprises to a greater extent data of epilepsy surgery and especially techniques than a neurosurgical textbook. The book addresses the intended readership, neurosurgeons and neurosurgeons in training. This book can be highly recommended to the neurosurgical community. 